\title{
Detailed Thermal Comfort Analysis from Preliminary to Final Design
}

\author{
Nathaniel L. Jones, Ingrid Chaires, and Alexej Goehring \\ Arup, San Francisco, USA
}

\begin{abstract}
Most thermal comfort models and indices consider the human body in environments with uniform and unchanging temperature. We developed a set of tools that simulate human response to changing and non-uniform thermal conditions adjacent to the body rather than average space conditions. This allows the analysis of thermal comfort under environmental conditions such as stratification, radiant asymmetry, and those created by personal environmental controls. We present a case study in which our tool is used to determine relaxed thermostat setpoints for multiple thermal zones of a US airport, leading to savings in energy consumption and first cost.
\end{abstract}

\section{Introduction}

Buildings are responsible for $39 \%$ of US energy use (EIA, 2018), and within commercial buildings, the combined demand of heating, ventilation, and air conditioning (HVAC) accounts for $35 \%$ of energy consumption (DOE, 2016). Load reduction strategies such as natural ventilation, wide thermostat setpoint bands, and personal comfort systems can reduce overall building energy demand, but they require careful analysis to avoid causing thermal discomfort to building occupants (Zhang, Arens, \& Zhai, 2015). Determining how best to leverage the external environment to reduce energy cost and maintain comfort requires tools that can describe and predict thermal sensation and comfort.

Currently, most tools and methods for assessing thermal comfort assume that environments are uniform and static in temperature. The ASHRAE 55 (2017) and ISO 7730 (2006) standards describe a range of thermal environments using the Predicted Mean Vote scale (Fanger, 1972), which assumes a homogeneous environment. Comfort standards such as these are slow to change and not necessarily representative of the modern workforce. Recent interest in personal comfort systems such as seat- or desk-mounted heaters and fans (Zhang, Arens, \& Zhai, 2015) or wearable devices (Delkumburewatte \& Dias, 2011) requires an investigation of comfort under non-uniform and timevarying conditions. Transient and asymmetric environments have a large bearing on thermal comfort but have received relatively little study, particularly in cases that are both changing and non-uniform. Common tools such as EnergyPlus (2018) and IES (2018) do not account for these conditions, and we are not aware of any commercial computational fluid dynamics or multiphysics packages with built-in thermal comfort tools. Furthermore, tools based on ASHRAE 55 or similar standards do not account for differences in build that affect individual perception of temperature.

In this paper, we develop a set of tools for predicting thermal sensation and comfort in time-varying and nonuniform environments. These tools include a web app for early design studies, a stand-alone application for detailed and customized studies, and a CFD plug-in for advanced multiphysics analysis, all based on a common software library. Routines in this library are validated against data collected from 27 individuals in tests at the University of California, Berkeley (Zhang, 2003). To demonstrate the usefulness of these tools, we present a case study of transient thermal comfort in a large airport. This design project includes multiple program elements with different comfort requirements and various user populations with different sensitivities moving through them. Our tools for advanced thermal comfort analysis allow us to improve comfort while reducing energy use and first cost.

\section{Background}

Most thermal comfort indices treat the body's temperature as constant and uniform. These indices typically calculate a uniform temperature under neutral conditions $(50 \%$ relative humidity and no air movement) at which the body's rate of heat exchange with its environment is the same as under actual conditions. These models may treat the body as a single node exchanging heat with its environment through some amount of insulation provided by clothing (Fanger, 1972) or they may divide the body into separate core and skin nodes so that the skin exchanges heat with both the core and environment (Gagge, Fobelets, \& Berglund, 1986). Some thermal comfort indices report the uniform environmental temperature directly, such as Standard Effective Temperature (SET*) (Gagge, Fobelets, \& Berglund, 1986), Equivalent Homogeneous Temperature (EHT) (Wyon, Larsson, Forsgren, \& Lundgren, 1989), and the Universal Thermal Climate Index for outdoor conditions (ISB, 2009). Others relate this temperature or the corresponding heat exchange rate to a subjective comfort scale. The Predicted Mean Vote (PMV) scale (Fanger, 1972) correlates heat exchange with a sensation scale from -3 (too cold) to 3 (too hot), with values between -1 and 1 indicating comfort. As a corollary, the Predicted Percentage Dissatisfied (PPD) scale relates the distance of 
the mean vote from neutral sensation to the fraction of the population that reports discomfort according to surveys (Fanger, 1972). Because all these indices assume constant and uniform thermal conditions, they register the greatest comfort in a neutral environment (neither warm nor cool).

\section{Advanced Thermal Comfort}

Advanced thermal comfort analysis accounts for human perception of variation in temperature around the body and over time. To do this, it is necessary to define separate scales for thermal sensation and thermal comfort. Sensation refers to the degree to which a person or body part feels hot or cold. Like the PMV scale, sensation varies from cold (negative numbers) to hot (positive numbers) with zero representing neutral temperature sensation (Zhang, Arens, Huizenga, \& Han, 2010a). Comfort refers to the level of satisfaction derived from the thermal environment. The comfort scale is similar to the sensation scale, but negative numbers represent dissatisfaction, while positive numbers correspond to positive satisfaction and zero represents indifference (Zhang, Arens, Huizenga, \& Han, 2010b). Notably, individual body parts may simultaneously experience different sensations. The comfort felt by each body part may be influenced by the sensations of other body parts, and each body has a different level of importance in determining overall sensation and overall comfort (Zhang, Arens, Huizenga, \& Han, 2010c).

Separating the concepts of sensation and comfort allows us to describe alliesthesia, the condition in which two opposing sensations result in greater comfort than either sensation can produce independently (Parkinson, de Dear, \& Candido, 2015). Opposite thermal sensations come in two forms. In asymmetric environments, simultaneous application of heating and cooling to different body parts can produce a pleasant overall sensation. In transient environments, an occupant moving from a cool space into a warm space (or vice versa) may take pleasure from the sudden removal of a thermal stress. Architectural precedents demonstrate the desirability of alliesthesial effects. In unconditioned homes, the hearth or k'ang provided comfort by warming one side of the body through radiation or conduction, while the frigidarium and caldarium of Roman baths allowed bathers to alternate between extreme thermal environments (Heschong, 1979).

\section{Physiology and Modelling}

Prediction of comfort in asymmetric and transient environments requires three steps. First, temperatures of individual body parts must be calculated as a time series. Second, local and overall sensations must be derived based on skin and core temperatures. Finally, comfort can be assigned to body parts and the whole in accordance with sensations.

Various models of human physiology and thermoregulation predict body part temperatures with different granularities. The Stolwijk (1971) model divides the body into ten cylindrical and spherical segments for the trunk, limbs, and head, each containing core, muscle, fat, and skin nodes with corresponding thermal capacitance in a nodal network. Heat is generated by metabolic production in each node and exchanged through the network between adjacent layers via conduction and with a central blood node representing convective heat transfer within the body (Shitzer, Arens, \& Zhang, 2015), as well as by convection and radiation to the environment (de Dear, Arens, Zhang, \& Oguro, 1997) and by latent diffusion through respiration and sweat (Stolwijk, 1971). Tanabe et al. (2002) expand Stolwijk's model to include 65 thermal nodes (four each in the head, chest, back, pelvis, upper and lower arms and legs, hands, and feet, in addition to the central blood node). Further refinements include modelling of non-cylindrical body parts (Ferreira \& Yanagihara, 2009), addition of arterial and vein nodes in each segment (Kobayashi \& Tanabe, 2013), and the division of each segment into differently oriented strips to account for radiant asymmetry (AlOthmani, Ghaddar, \& Ghali, 2008). To support this, thermal manikin studies have been used to collect clothing insulation (Lee, Zhang, \& Arens, 2013) and radiant and convective heat transfer coefficients for each body segment (de Dear, Arens, Zhang, \& Oguro, 1997; Oguro, Arens, de Dear, Zhang, \& Katayama, 2002).

Only a few studies directly relate individual body part temperatures to overall sensation and comfort. Most of these are modifications of PMV or SET*. Asymmetric thermal environments have been considered in the automotive industry, where directed airstreams can easily serve distinct regions of the body (Hagino \& Hara, 1992; Ingersoll, Kalman, Maxwell, \& Niemiec, 1992; Brown \& Jones, 1997; Kohri \& Mochida, 2003). Sensation under transient conditions has also been assessed for uniform environments (Wang \& Peterson, 1992; Fiala, Lomas, \& Stohrer, 2003). In a series of papers, Zhang et al. (2010a; 2010b; 2010c) developed a comprehensive model for sensation and comfort that separately considers 19 body parts (dividing the head from the Tanabe model into face, head, and neck, and adding a breathing zone for the inside of the mouth) under both transient and asymmetric conditions. Following work by Frank et al. (1999), Zhang et al. find that local sensation is influenced equally by skin and core temperatures, as well as by the rate of heat gain or loss:

$$
\text { Local Sensation }=f\left(T_{\text {skin }}, \frac{d T_{\text {skin }}}{d t}, T_{\text {core }}, \frac{d T_{\text {core }}}{d t}\right)
$$

The body's overall sensation is calculated using one of seven models, depending on whether any body parts experience unpleasant sensations (hot or cold, rather than warm or cool) or sensations opposite the dominant sensation (hot while the rest of the body is cool, or vice versa) (Zhang, Arens, Huizenga, \& Han, 2010c). Local comfort is highest when body parts are close to neutral sensation or act to pull the overall sensation away from an extreme. Overall comfort is determined by the group of body parts with the greatest local discomforts. To express these values, Zhang et al. developed numerical scales inspired by the PMV scale. Sensation values range from -4 (very cold) to 4 (very hot), and comfort from -4 (very uncomfortable) to 4 (very comfortable). 


\section{Method}

Our approach to predicting thermal comfort is to calculate skin and core temperatures using the 65-node human physiological model (Tanabe, Kobayashi, Nakano, Ozeki, \& Konishi, 2002) and apply these as inputs to the thermal sensation and comfort models by Zhang et al. (2010a; 2010b; 2010c). To do this, we created a set of tools built around a single software library that implements these models in an object-oriented manner. In this section, we describe the common software library and three tools intended for use at different stages of design that implement this library.

Regardless of how the tool is used, the software library requires the same input data to describe a list of static or transient environments. Each environment, or simulation phase, requires the following input:

- Length of the phase in minutes

- Dry bulb temperature

- Mean radiant temperature

- Relative humidity

- Air velocity

- Metabolic rate

- Clothing ensemble description

The temperatures, humidity, and air velocity may be provided separately for the air surrounding each of the 16 body parts or as single values if the conditions are uniform. The library performs an iterative simulation in which a body encounters each of the phases for the specified length of time and produces the following time series as output:

- Local skin temperatures

- Skin wettedness from sweat

- Temperature and humidity of respiratory air

- Local and overall sensations

- Local and overall comfort levels

- Additional comfort indices:
○ Standard Effective Temperature (SET*)
○ Effective Temperature (ET*)
- Discomfort Index (DISC)
○ Thermal Sensation Index (TSENS)
- Predicted Mean Vote (PMV)
○ Predicted Percentage Dissatisfied (PPD)

While the software library generally follows the works of Tanabe and Zhang, we have made several improvements.

- We adjust certain physiological parameters based on height, weight, and body composition, if these are known. The body surface area, used to compute a variety of heat production and heat flow terms, is set based on height and weight (Du Bois \& Du Bois, 1916). The body's thermal capacitance and metabolic heat production are adjusted proportionally to weight, with additional modification if the fat content of the body is known (Hodgdon \& Beckett, 1984a; Hodgdon \& Beckett, 1984b).

- We adjust the convective heat transfer coefficients according to air speed (de Dear, Arens, Zhang, \&
Oguro, 1997). The input air velocity may also be used to represent motion of the body through still air.

- We introduce a maximum simulation time step to ensure smooth derivatives in the physiology model output. The default for this user-adjustable setting is 10 seconds. This value is close to the reporting frequency of sensors in Zhang's tests, so phase lengths less than this setting are not recommended.

- When a condition is encountered for the first time in a simulation, we perform a warm-up routine to establish neutral sensation set-points. We first calculate the uniform temperature that achieves a PMV of zero under current air speed, air moisture content, metabolic rate, and overall clothing insulation, and we then allow the 65-node model to coast under these conditions until it reaches a steady state, as suggested by Zhao et al. (2014). The individual body parts' skin temperatures at steady state become the neutral setpoints for use in Zhang's local sensation model.

- We adjust the local sensation of each body part to account for age, sex, and fat content of individuals (Zhang, 2003).

- Rather than calculate only the relevant one of Zhang's seven overall sensation models, we calculate all of them and use sigmoid smoothing functions to interpolate between them, as suggested by Zhao et al. (2014). This removes discontinuities from the time series of overall sensation that occur when the relevant model changes. Furthermore, we simplify the control logic involved in selecting the dominant overall sensation model from what Zhang et al. (2010c) originally proposed without affecting the outcome of the calculation (Figure 1).

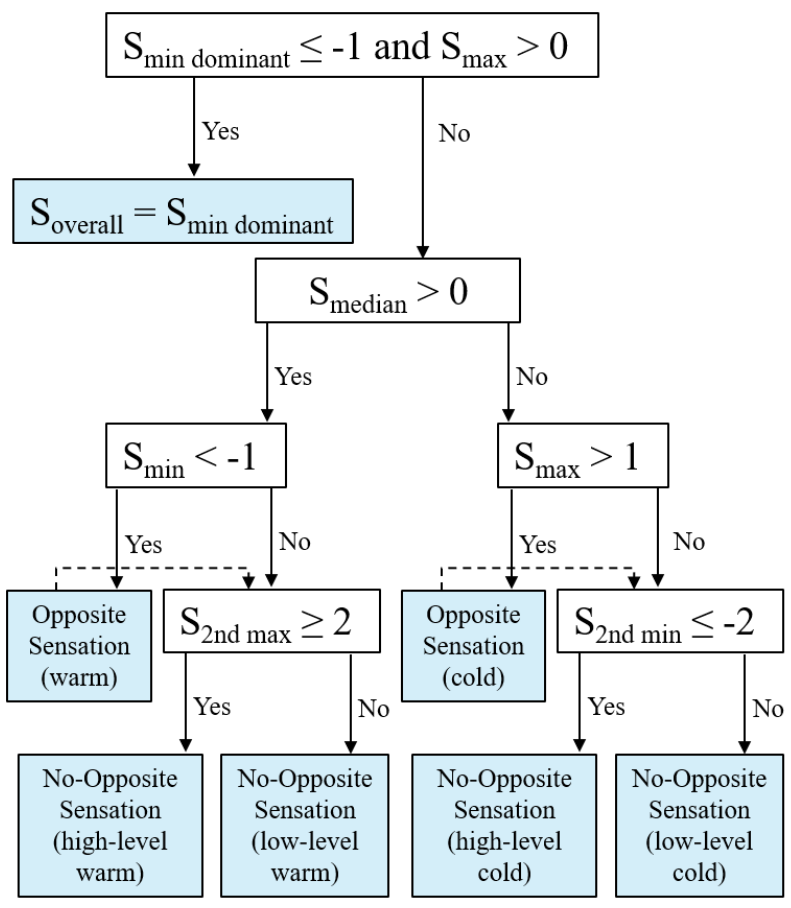

Figure 1: Control logic for selecting one of Zhang's seven overall sensation models (shaded boxes) based on the array $\mathrm{S}$ of local body part sensations. 
- Zhang's overall sensation model for asymmetrical conditions defines an individual force for each body part that quantifies its influence on overall sensation (Zhang, Arens, Huizenga, \& Han, 2010c). Each individual force is a function of the change in local sensation since the beginning of the heating or cooling period. In order to apply this model to scenarios in which the body is exposed to multiple transient environments in sequence without abrupt changes in the reference sensations, we store a history of local sensations and calculate individual forces at each time step in reference to sensations recorded a certain amount of time earlier. This creates a moving window of thermal sensation "memory" that influences the current sensation.

With the software library we have described, we created three tools for evaluating thermal sensation and comfort. We developed each tool for use at a specific design phase, although all tools may be used at any point during design.

\section{Web Tool}

The web tool is intended for early design studies. The goal of thermal comfort studies in early design is to establish temperature, humidity, and air velocity requirements to ensure that the design will provide comfortable conditions. The interface (Figure 2) allows the user to rapidly define and edit environments by giving each a name, dry bulb and mean radiant temperatures, relative humidity, and air velocity. The user can arrange these environments to form a path in which each environment may appear repeatedly, and the user can set the time duration spent in each environment and the activity level and clothing ensemble worn in each phase. The simulation runs immediately on any change to the input, allowing the user to make informed decisions with immediate and continuous feedback. No extra step is required to start a simulation.

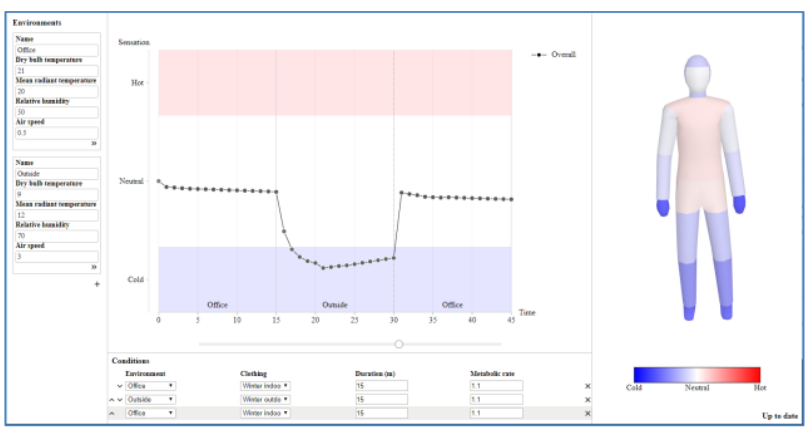

Figure 2: The web tool allows input of thermal a series of thermal environments (left side) and display of resulting sensation and comfort levels (right side).

The centre of the web tool interface contains a time history plot showing overall sensation or comfort, together with the local sensation or comfort of any selected body parts. Next to this, a manikin displays colorcoded thermal sensations for all body parts. A time slider under the plot controls the moment in time displayed on the manikin. Any change to the environment or path inputs is immediately reflected in the output display.

\section{Stand-Alone Application}

The stand-alone application is a command-line utility intended for more detailed and customized studies than are possible with the simplified web interface. This tool can model any transient or asymmetric condition per the designer's intent. It requires the user to generate an input file in comma separated value (CSV) format listing conditions for all environments in sequence. It produces output temperatures, sensations, comfort values, and other indices also in CSV format. While the stand-alone application may be accessed directly by the user, it also offers maximum flexibility for scripting. It can be called on by DOS, Python, Grasshopper, or any other scripting language, and scripting tools may also be used to automatically generate the input CSV file and to interpret and display the output.

\section{CFD Plug-In}

Our CFD plug-in is geared toward multiphysics analysis which typically occurs later in design or as part of nonstandard design projects. For transient computational fluid dynamics (CFD) simulations, the plug-in allows a manikin modelled in the CFD domain to give off heat and moisture to its surroundings as directed by the physiological model in our software library (Figure 3). At each simulation timestep, the dry bulb and mean radiant temperature, relative humidity, and air velocity around each body part are read from the CFD simulation, and a similar time step iteration is performed using our library to calculate new skin temperatures, skin wettedness, and respiratory heat and moisture that feed back into the CFD simulation as updated boundary conditions. The plug-in also allows local and overall sensation and comfort values to be collected by the CFD tool and output as time series or final values at the end of the simulation. Currently, the plug-in targets ANSYS CFX (2018), but this serves as a proof of concept toward eventual broader applicability.

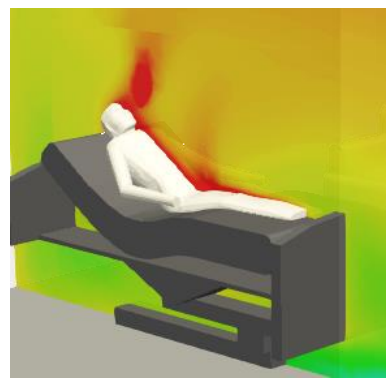

Air Temperature $\left({ }^{\circ} \mathrm{F}\right)$

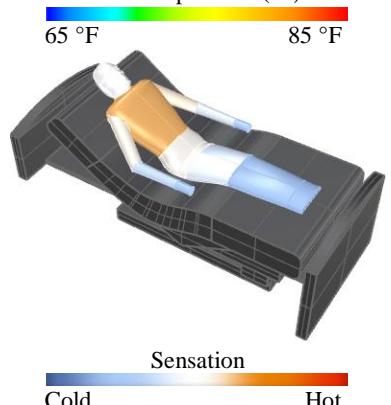

Figure 3: The CFD plug-in causes environmental conditions such as temperature and humidity (top) to interact with the body's thermal experience (bottom). 

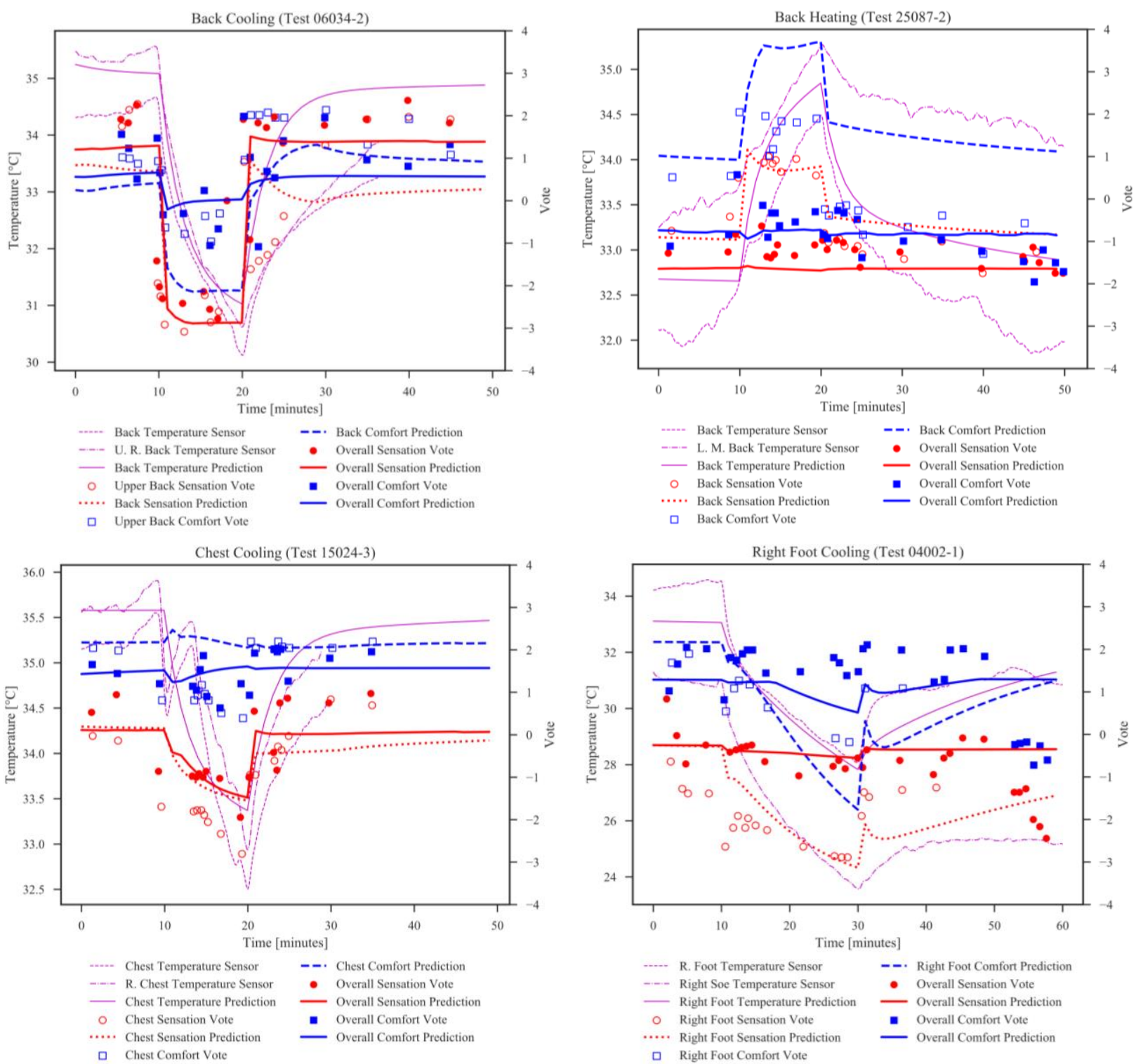

Figure 4: Correspondence between measured and predicted skin temperature, sensation, and comfort for selected tests of local body part cooling or heating.

\section{Validation}

We verified the accuracy of our software library by simulating conditions from some of the human subject tests originally used by Zhang (2003) to develop the sensation and comfort models. Zhang performed 109 tests on 15 female and 12 male volunteers. Tests involved cooling or heating one or more body parts while the participants reported thermal sensation and comfort via an electronic survey. Thermocouples worn under clothing recorded skin temperatures, and subjects swallowed a radio pill to record core temperature. Typical tests included multiple heating or cooling phases separated by a recovery period to return to ambient conditions.

We recreated the environmental conditions for each test as input to our stand-alone tool and compared the predicted skin temperatures, sensations, and comfort levels to original data collected by Zhang (Figure 4). Because Zhang did not record the exact start and end times for each cooling or heating phase, we created an automated script to identify these phases from the recorded data and align our simulations with the timestamps on the original data. This was effective with local cooling tests, but it proved more difficult with local heating tests because changes in skin temperature in response to local heating were often less apparent. However, subjects also reported less discomfort from local heating, so our main concern is with predicting the effects of cooling the body.

In the tests shown in Figure 4, most of our predictions track Zhang's recordings within about one degree for temperature and one unit on the voting scales for sensation and comfort. Our simulations reflect Zhang's observations that sensation changes suddenly upon exposure to a new environment, while skin temperature adjusts more gradually, and that central body parts affect overall sensation and comfort more strongly than extremities. In general, our model performs better on core body parts such as the back than on extremities such as 
Table 1: Airport passenger path.

\begin{tabular}{|c|c|c|c|c|c|}
\hline Zone & $\begin{array}{c}\text { Time } \\
(\text { minutes) }\end{array}$ & Activity & $\begin{array}{c}\text { Metabolic } \\
\text { Rate (MET) }\end{array}$ & Baseline Setpoint $\left({ }^{\circ} \mathbf{F}\right)$ & $\begin{array}{c}\text { Adjusted Operative } \\
\text { Temperature Setpoints }\left({ }^{\circ} \mathbf{F}\right)\end{array}$ \\
\hline Entry & 15 & Walking & 1.4 & 72 & $62-82$ \\
\hline Ticketing & 15 & Walking, Standing & 1.2 & 72 & $66-80$ \\
\hline Security & 15 & Standing & 1 & 72 & $68-76$ \\
\hline Concessions & 30 & Walking, Standing, Sitting & 1.1 & 72 & $62-82$ \\
\hline Hold Room & 45 & Sitting & 1 & 72 & $70-74$ \\
\hline
\end{tabular}

the head and hands. This may be because Zhang treated the head as multiple zones, differentiating the face, scalp, and neck, and also because rapid vasodilation and vasoconstriction in the hands result in more varied skin temperatures than predicted by Tanabe, et al. (2002).

\section{Case Study}

Our tool was used to plan the thermal comfort setpoints for multiple thermal zones in an airport expansion project located in the Pacific Northwest. Airport design is complex due to the variety of occupants, space types, and dwell times in those spaces. This makes airports interesting and difficult to analyse for thermal comfort.

Passengers experience transient effects as they take different paths through an airport. A common path is entering the airport, going to the ticketing kiosks, waiting in line and passing through security, shopping and dining in the concession area, and finally waiting at the boarding gate. This path takes a passenger from thermal conditions of the outdoors to those of the hold room where passengers could have a dwell time on the order of hours. Designers must consider the activity and dwell times of the passengers as they progress through the building when developing a thermal zoning strategy.

Thermal considerations differ for the staff. Airport staff typically have longer dwell times in the various thermal zones. Therefore, specific systems need to be designed to meet the thermal comfort needs of the staff who experience their local thermal environment for longer periods of time than the passengers do. Localized or personal controls can serve the stricter needs of staff, while the main conditioning systems serve passengers.

A large airport can be split into many thermal zones with different cooling and heating loads and different comfort criteria. Airport HVAC systems can be chosen to meet these loads and achieve thermal comfort. There is a myriad of available system types and distribution strategies, depending on the space in question. The chosen HVAC systems need to meet adequate thermal comfort criteria and also meet energy efficiency design goals.

Here, we look at one path that a passenger might take through entry, ticketing, security, and concessions to the final hold room. The time spent for each space is broken down in Table 1 with the original and proposed setpoints to achieve thermal comfort for each space. Our earlystage modelling assumes $50 \%$ relative humidity and still air with average velocity $0.1 \mathrm{~m} / \mathrm{s}(20 \mathrm{fpm})$. Occupants are expected to dress in typical summer clothes (0.5 CLO) and winter indoor clothes (1.0 CLO) on dates at the upper and lower ends of the adjusted setpoint band, respectively.
We propose the adjusted setpoints in Table 1 such that the transient thermal sensation of passengers on this path will remain neutral. Our tools shows no thermal discomfort resulting from the adjusted temperature setpoints under the summer (Figure 5) or winter (Figure 6) conditions in our model. Therefore, our recommendation was to use this expanded temperature range during operations.

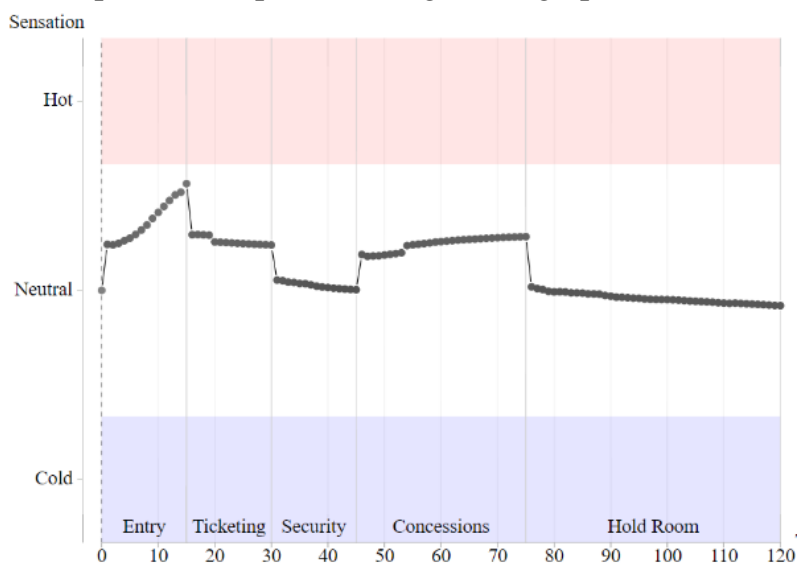

Figure 5: Thermal sensation for a typical passenger path in an airport on a summer day.

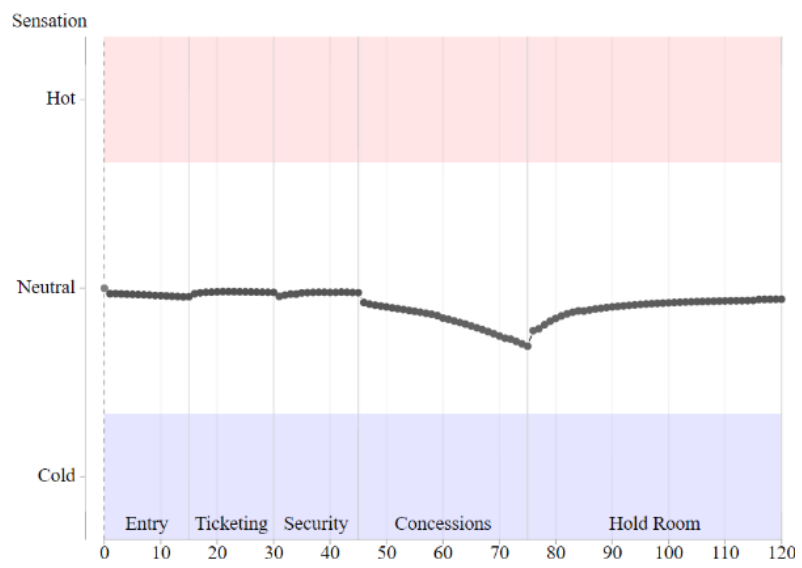

Figure 6: Thermal sensation for a typical passenger path in an airport on a winter day.

Relaxing the setpoints reduces HVAC system energy consumption in building operation. We used EnergyPlus to model annual energy use in the spaces listed in Table 1. Figure 7 compares the expanded setpoints to a baseline model with a constant temperature setpoint of $72^{\circ} \mathrm{F}$ for all spaces. The expanded setpoints cause a reduction in annual heating energy use of $38 \%$ and a $26 \%$ savings in annual cooling energy. The reductions in HVAC energy use show the operational changes can save energy without adverse effects to overall thermal comfort. 


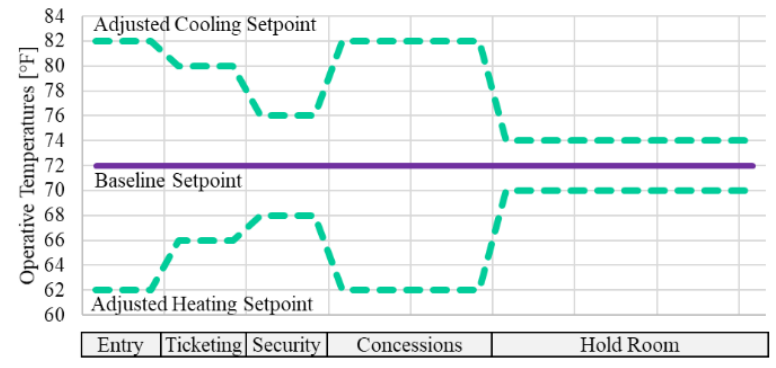

Figure 7: Baseline temperature setpoint vs adjusted temperature setpoints by space and dwell time.

In addition to energy savings during building operation, there is also a reduction in first cost of the HVAC equipment as the system sizes are decreased. Therefore, expanded setpoints can save on both the first cost of HVAC equipment and energy during building operation, while providing comfort to the airport passengers.

\section{Discussion}

Providing thermal comfort is an important function of buildings and a major reason for the use of HVAC systems. The tools we describe in this paper can give designers valuable insight into the thermal comfort implications of transient and non-uniform environments. Accounting for transient effects can lead to expanded setpoint temperatures that save energy during the building operation and reduce equipment first cost. Our tools can also quantify the effects of other energy and sustainability measures such as personal control systems, passive systems, and task HVAC controls.

The validation presented in this paper represents a limited dataset provided by the Center for the Built Environment. We closely replicated the conditions from Zhang's study. However, thermal comfort continues to be highly subjective, and it is not our aim to predict an individual's thermal comfort. Rather, thermal models must grow to encompass groups and populations, accounting for variation in individual preference and physiology.

Future work should focus on validating the tool's predictions against population data and integrating thermal comfort data into design workflows. We hope for tools such as ours to become commonplace in the design industry and for practitioners to acknowledge thermal comfort analysis as a necessary input for design.

\section{Conclusion}

We developed tools that predict thermal comfort under transient and asymmetric conditions for use at multiple design phases. These include a web tool aimed at early design, a stand-alone application for detailed analysis, and a CFD plug-in for multiphysics analysis of non-standard projects. The tools are based on a shared software library which was able to predict skin temperatures within one degree and thermal sensation and comfort within one voting scale increment for selected validation test cases.

We applied the tool to an airport project to demonstrate the usefulness of an expanded setpoint band for multiple different thermal zones to reduce energy consumption and HVAC equipment first cost. Our software revealed that a wider thermostat setpoint band can maintain comfort and reduce energy consumption by up to $38 \%$ in winter and $26 \%$ in summer. This is in keeping with our broader goal to create a positive building user experience by making simulation data available at all stages of design.

\section{Acknowledgment}

The authors gratefully acknowledge Hui Zhang, Edward Arens, Charlie Huizenga, and David Lehrer at the Center for the Built Environment. Ian Mackenzie developed the web tool interface. Software development was funded by Invest in Arup projects 16122, 18517, and 22229.

\section{References}

Al-Othmani, M., Ghaddar, N., \& Ghali, K. (2008). A multi-segmented human bioheat model for transient and asymmetric radiative environments. International Journal of Heat and Mass Transfer, 51, 5522-5533.

ANSYS. (2018). CFX 19.1.

ASHRAE. (2017). ASHRAE/ANSI Standard 55-2017 Thermal environmental conditions for human occupancy. Atlanta (USA): American Society of Heating, Refrigerating, and Air-Conditioning Engineers.

Brown, J. S., \& Jones, B. W. (1997). A new transient passenger thermal comfort model. SAE Technical Paper 970528.

de Dear, R. J., Arens, E., Zhang, H., \& Oguro, M. (1997). Convective and radiative heat transfer coefficients for individual human body segments. International Journal of Biometeorology, 40(3), 141-156.

Delkumburewatte, G. B., \& Dias, T. (2011). Wearable cooling system to manage heat in protective clothing. The Journal of The Textile Institute, 103(5), 483-489.

DOE. (2016). Quadrennial technology review: An assessment of energy technologies and research opportunities. Washington DC (USA): U.S. Department of Energy.

Du Bois, D., \& Du Bois, E. (1916). A formula to estimate the approximate surface area if height and weight be known. Archives of Internal Medicine, 17(6), 863871.

EIA. (2018). December 2018 Monthly Energy Review. Washington DC (USA): U.S. Energy Information Administration.

EnergyPlus Development Team. (2018). EnergyPlus engineering reference: The reference to EnergyPlus calculations. EnergyPlus Version 9.0: US Department of Energy.

Fanger, P. O. (1972). Thermal comfort: Analysis and applications in environmental engineering. New York (USA): McGraw-Hill Book Company.

Ferreira, M., \& Yanagihara, J. (2009). A transient threedimensional heat transfer model of the human body. International Communications in Heat and Mass Transfer, 36, 718-724. 
Fiala, D., Lomas, K. J., \& Stohrer, M. (2003). first principles modeling of thermal sensation responses in steady-state and transient conditions. ASHRAE Transactions, 109(1), 179-186.

Frank, S. M., Raja, S. N., Bulcao, C. F., \& Goldstein, D. S. (1999). Relative contribution of core and cutaneous temperatures to thermal comfort and autonomic responses in humans. Journal of Applied Physiology, 86(5), 1588-1593.

Gagge, A., Fobelets, A., \& Berglund, L. (1986). A standard predictive index of human response to the thermal environment. ASHRAE Transactions, 92, 709-731.

Hagino, M., \& Hara, J. (1992). development of a method for predicting comfortable airflow in the passenger compartment. SAE Technical Paper 922131. doi:10.4271/922131

Heschong, L. (1979). Thermal Delight in Architecture. Cambridge (USA): MIT Press.

Hodgdon, J. A., \& Beckett, M. B. (1984a). Prediction of percent body fat for U.S. Navy men from body circumferences and height. San Diego (USA): Naval Health Research Center.

Hodgdon, J. A., \& Beckett, M. B. (1984b). Prediction of percent body fat for U.S. Navy women from body circumferences and height. San Diego (USA): Naval Health Research Center.

IES. (2018). IES VE 2018.

Ingersoll, J. G., Kalman, T. G., Maxwell, L. M., \& Niemiec, R. J. (1992). Automobile passenger compartment thermal comfort model — part II: Human thermal comfort calculation. SAE Transactions.

ISB Commission 6. (2009). Development of a "Universal Thermal Climate Index" (UTCI). International Society of Biometeorology.

ISO 7730. (2006). Ergonomics of the thermal environment - Analytical determination and interpretation of the thermal comfort using calculation of the PMV and PPD indicies and local thermal comfort criteria. Geneva (Switzerland): International Organization for Standardization.

Kobayashi, Y., \& Tanabe, S.-i. (2013). Development of JOS-2 human thermoregulation model with detailed vascular system. Building and Environment, 66, 1-10.

Kohri, I., \& Mochida, T. (2003). Evaluation method of thermal comfort in a vehicle with a dispersed twonode model part 2 - Development of new evaluation. Journal of the Human-Environment System, 6(2), 77-91.

Lee, J., Zhang, H., \& Arens, E. (2013). Typical clothing ensemble insulation levels for sixteen body parts. Proceedings of the 11th International Conference CLIMA 2013. Prague (Czech Republic).

Oguro, M., Arens, E., de Dear, R. J., Zhang, H., \& Katayama, T. (2002). Convective heat transfer coefficients and clothing insulations for parts of the clothed human body under airflow conditions. Journal of Architecture and Planning, 67(561), 21-29.

Parkinson, T., de Dear, R., \& Candido, C. (2015). Thermal pleasure in built environments: Alliesthesia in different thermoregulatory zones. Building Research \& Information, 44(1), 20-33.

Shitzer, A., Arens, E., \& Zhang, H. (2015). Compilation of basal metabolic and blood perfusion rates in various multi-compartment, whole-body thermoregulation models. International Journal of Biometeorology, 60(7), 1051-1064.

Stolwijk, J. (1971). A mathematical model of physiological temperature regulation in man. Washington DC (USA): NASA CR-1855.

Tanabe, S.-i., Kobayashi, K., Nakano, J., Ozeki, Y., \& Konishi, M. (2002). Evaluation of thermal comfort using combined multi-node thermoregulation (65MN) and radiation models and computational fluid dynamics (CFD). Energy and Buildings, 34(6), 637646.

Wang, X. L., \& Peterson, F. K. (1992). Estimating thermal transient comfort. ASHRAE Transactions, 98, 182-188.

Wyon, D., Larsson, S., Forsgren, B., \& Lundgren, I. (1989). Standard procedures for assessing vehicle climate with a thermal manikin. SAE Technical Paper 890049.

Zhang, H. (2003). Human thermal sensation and comfort in transient and non-uniform thermal environments. PhD Thesis: University of California, Berkeley.

Zhang, H., Arens, E., \& Zhai, Y. (2015). A review of the corrective power of personal comfort systems in nonneutral ambient environments. Building and Environment, 91, 15-41.

Zhang, H., Arens, E., Huizenga, C., \& Han, T. (2010a). Thermal sensation and comfort models for nonuniform and transient environments: Part I: Local sensation of individual body parts. Building and Environment, 45(2), 380-388.

Zhang, H., Arens, E., Huizenga, C., \& Han, T. (2010b). Thermal sensation and comfort models for nonuniform and transient environments, part II: Local comfort of individual body parts. Building and Environment, 45(2), 389-398.

Zhang, H., Arens, E., Huizenga, C., \& Han, T. (2010c). Thermal sensation and comfort models for nonuniform and transient environments, part III: Wholebody sensation and comfort. Building and Environment, 45(2), 399-410.

Zhao, Y., Zhang, H., Arens, E. A., \& Zhao, Q. (2014). Thermal sensation and comfort models for nonuniform and transient environments, part IV: Adaptive neutral setpoints and smoothed whole-body sensation model. Building and Environment, 72, 300-308. 